\title{
ON THE RESTRICTION OF THE FOURIER TRANSFORM TO CURVES: ENDPOINT RESULTS AND THE DEGENERATE CASE
}

BY

MICHAEL CHRIST ${ }^{1}$

\begin{abstract}
For smooth curves $\Gamma$ in $\mathbf{R}^{n}$ with certain curvature properties it is shown that the composition of the Fourier transform in $\mathbf{R}^{n}$ followed by restriction to $\Gamma$ defines a bounded operator from $L^{p}\left(\mathbf{R}^{n}\right)$ to $L^{q}(\Gamma)$ for certain $p, q$. The curvature hypotheses are the weakest under which this could hold, and $p$ is optimal for a range of $q$. In the proofs the problem is reduced to the estimation of certain multilinear operators generalizing fractional integrals, and they are treated by means of rearrangement inequalities and interpolation between simple endpoint estimates.
\end{abstract}

1. Introduction. Given a smooth submanifold $M$ of $\mathbf{R}^{n}$ and a smooth positive measure $\sigma$ on $M$ one may ask for which exponents $p$ and $q$ there is a restriction estimate

$$
\int_{M}|\hat{f}(\xi)|^{q} d \sigma \leq C\|f\|_{L^{p}\left(\mathbf{R}^{n}\right)}^{q}
$$

for the Fourier transform of an arbitrary test function $f \in S\left(\mathbf{R}^{n}\right) . \hat{f}$ denotes the Fourier transform, and $S$ the class of Schwartz functions. Interest in this problem is due largely to its intimate connections with Bochner-Riesz multipliers $[\mathbf{3}, \mathbf{9}, \mathbf{1 2}]$ and certain estimates for wave equations [17]. The first topic of this article is the case when $M$ is a curve, say $M=\{\psi(t):|t| \leq 1\}$ where $\psi:[-1,1] \rightarrow \mathbf{R}^{n}$ is $C^{\infty}$. The curve is said to be of finite type at $t=0$ if $\left\{\psi^{\prime}(0), \psi^{\prime \prime}(0), \ldots \psi^{(l)}(0)\right\}$ spans $\mathbf{R}^{n}$ for some $l$; it is nondegenerate at 0 if it is of finite type and $l=n$. By a well-known homogeneity argument $[\mathbf{1 9}]$,

$$
\int_{-\delta}^{\delta}|\hat{f}(\psi(t))|^{q} d t \leq C\|f\|_{p}^{q}
$$

can hold for some $0<\delta$ and $1<p$ only if the curve is of finite type at $t=0$. If $0=$ $k_{1}<k_{2}<\cdots<k_{n}$ are positive integers, we say that $\psi$ is of type $k=\left(k_{1}, \ldots, k_{n}\right)$ at $t=0$ if it is possible to make a linear change of coordinates in $\mathbf{R}^{n}$ and a nonsingular reparametrization of $(-1,1)$ to bring $\psi$ into the form $\psi(t)=\left(t, \psi_{2}(t), \ldots, \psi_{n}(t)\right)$, where $\psi_{j}^{\prime}(t)=t^{k_{j}}(1+O(t))$. (Note that under these conventions the nondegenerate case is $k=(0,1, \ldots, n-1)$.)

Received by the editors July 7, 1983 and, in revised form, January 3, 1984.

1980 Mathematics Subject Classification. Primary 42B10, 26D10.

Key words and phrases. Fourier transform, curvature, multilinear operator, interpolation.

${ }^{1}$ Supported in part by NSF grant No. MCS- 8211327. 
Our principal result for curves is then

ThEOREM 1.1. Suppose $\psi:(-1,1) \rightarrow \mathbf{R}^{n}$ is smooth with $\psi^{\prime} \neq 0$, of finite type $k$ at $t=0$. Then there exists $\delta>0$ such that

$$
\int_{-\delta}^{\delta}|\hat{f}(\psi(t))|^{q} d t \leq C\|f\|_{p}^{q}
$$

for all $f \in S\left(\mathbf{R}^{n}\right)$ if:

(A) $\psi$ is nondegenerate at $t=0$.

$$
(n+|k|)\left(1-p^{-1}\right) \leq q^{-1} \quad \text { and } \quad 1 \leq p<\frac{n^{2}+2 n}{n^{2}+2 n-2}=\frac{n^{2}+|k| n-n}{n^{2}+|k| n-2 n+1}
$$

or

(B) $\psi$ is degenerate at $t=0$ and either

$$
(n+|k|)\left(1-p^{-1}\right) \leq q^{-1} \quad \text { and } \quad 1 \leq p \leq \frac{n^{2}+|k| n-n}{n^{2}+|k| n-2 n+1},
$$

or

$$
(n+|k|)\left(1-p^{-1}\right)<q^{-1} \quad \text { and } \quad \frac{n^{2}+|k| n-n}{n^{2}+|k| n-2 n+1}<p<\frac{n^{2}+2 n}{n^{2}+2 n-2} .
$$

Here $|k|=\sum_{1}^{n} k_{j}$.

The restriction $(n+|k|)\left(1-p^{-1}\right) \leq q^{-1}$ is necessary by the usual homogeneity argument $[\mathbf{1 8}, \mathbf{1 9}]$, but whether the restrictions on the range of $p$ and the strict inequality in (1.3) are necessary is not known. In the nondegenerate case only the endpoint result $\frac{1}{2} n(n+1)\left(1-p^{-1}\right)=q^{-1}$ is new $[\mathbf{1 4}]$. One motivation for studying the endpoint situation is the case of certain noncompact curves: an immediate consequence of Theorem 1.1 is

COROLLARY 1.2. Let $1=l_{1}<l_{2}<\cdots<l_{n}$ be positive integers and $l=\sum_{1}^{n} l_{j}$. Then

$$
\int_{-\infty}^{\infty}\left|\hat{f}\left(t^{l_{1}}, \ldots, t^{l_{n}}\right)\right|^{q} d t \leq C\|f\|_{p}^{q}
$$

for all $f \in S\left(\mathbf{R}^{n}\right)$ if $p^{\prime}=l q$ and either

$$
l_{n}=n \quad \text { and } \quad 1 \leq p<\frac{n l-n}{n l-2 n+1}=\frac{n^{2}+2 n}{n^{2}+2 n-2}
$$

or

$$
l_{n}>n \quad \text { and } \quad 1 \leq p \leq \frac{n l-n}{n l-2 n+1} \quad\left(<\frac{n^{2}+2 n}{n^{2}+2 n-2}\right) .
$$

Now the stipulation $p^{\prime}=l q$ is necessary, again by homogeneity; only in the endpoint case is there a restriction estimate.

Previously the degenerate case had not been studied extensively. Partial results in the special case $n=3$ and $k_{2}=1$ were obtained by Ruiz [15].

The strategy of our proof is not new: following Prestini, we ultilize an argument originating in Fefferman $[\mathbf{9}]$ and Carleson and Sjölin $[\mathbf{3}]$, based on a change of variables and the Hausdorff-Young inequality, to reduce (1.1) to an easier problem concerning estimates for certain positive integral operators. When $n=2$ and $M$ 
has nonvanishing curvature, these are the classical fractional integration operators, and endpoint estimates for (1.1) follow from their well-known mapping properties. But for nondegenerate curves in dimension 3 or more the operators which arise in this line of argument had only been treated in a relatively crude way by means of Hölder's inequality [14], so that endpoint estimates were not obtained. Our only innovation is to view these operators as multilinear versions of fractional integral operators and to prove optimal estimates for them. This is done in the second section of the paper.

While the various technical hypotheses of Theorem 1.1 are not known to be necessary and appear rather unnatural, they arise inevitably from the method of proof. In $\S 2$ we also show that the multilinear operators to which the problem "reduces" are actually unbounded in all other instances. Nevertheless our counterexamples fail to provide counterexamples for the restriction problem itself; one can conclude only that the present line of attack cannot resolve these remaining issues.

Our second topic is the case of manifolds of dimension 2 in $\mathbf{R}^{4}$ satisfying a suitable nondegeneracy condition. Prestini $[\mathbf{1 4}]$ has studied the restriction problem for manifolds of half the dimension of the ambient space, but again without obtaining endpoint results. The primary motivation for our interest in this special case is the study [4], where the author has investigated, but not completely settled, a general conjecture for manifolds of codimension two. One way to view this conjecture is as a statement that a certain curvature condition is the "right" notion of nondegeneracy for the restriction problem in codimension two. The case of total dimension four is exceptional, for then the dimension of the ambient space is an integral multiple of the dimension of the submanifold. This enables us to prove the conjecture by the same methods as employed for curves in previous sections of the paper.

In $\S 4$ we discuss a variant of a theorem of Helson and Beurling which is related to the restriction problem, and our final subject is a certain family of multilinear singular integral operators which complement the "fractional integral" operators treated in $\S 2$.

I would like to thank W. Beckner and L. Carleson for pointing out the relevance of rearrangement inequalities, which led to a shortened proof of Proposition 2.3. Thanks also to L. Carleson and P. W. Jones of the Institut Mittag-Leffler for their hospitality during the final stages of this work, and to the referee for suggestions regarding the exposition.

2. Multilinear operators and restriction to curves. To commence with the reduction of Theorem 1.1 to a question about multilinear operators, consider the formal adjoint of the "restriction operator". Up to a constant factor it is

$$
A f(\xi)=\int_{-\delta}^{\delta} \exp (i\langle x(t), \xi\rangle) f(t) d t
$$

A surprisingly helpful step, ultimately because of Lemma 2.1 , is to assume that $f$ is supported where $t \geq 0$. The case where support $(f) \subset\{t \leq 0\}$ is no different, so it may be disregarded. Thus

If $p^{\prime} \geq 2 n$ then

$$
A f(\xi)=\int_{0}^{\delta} \exp (i\langle\psi(t), \xi\rangle) f(t) d t
$$

$$
\|A f\|_{p^{\prime}}^{n}=\left\|(A f)^{n}\right\|_{p^{\prime} / n}
$$


and the point is that $(A f)(\xi)^{n}$ may be written as a Fourier transform:

$$
(A f(\xi))^{n}=\int \exp \left(i\left\langle\xi \cdot \sum_{1}^{n} \psi\left(x_{i}\right)\right\rangle\right) \prod_{1}^{n} f\left(x_{i}\right) d x,
$$

where $x=\left(x_{1} \ldots x_{n}\right) \in \mathbf{R}^{n}$ and $0 \leq x_{i} \leq \delta$. Formally change variables by setting $y_{j}=\sum_{1}^{n} \psi_{j}\left(x_{i}\right)$ : this will be justified below. Then

$$
A f(\xi)^{n}=\int \exp (i\langle\xi, y\rangle) F(y) d y=\check{F}(\xi)
$$

where $F(y)=\prod f\left(x_{i}\right)|\partial x / \partial y|$. By the inequality of Hausdorff and Young

$$
\|A f\|_{p^{\prime}}^{n} \leq c\|F\|_{r}
$$

where $r^{\prime}=p^{\prime} / n$. Reversing the change of variables,

$$
\|F\|_{r}^{r}=\int_{0 \leq x_{1} \ldots x_{n} \leq \delta}\left|\prod f\left(x_{i}\right)\right|^{r}\left|\frac{\partial y}{\partial x}\right|^{1-r} d x .
$$

The nature of the singularities of the integral kernel $|\partial y / \partial x|^{1-r}$ is thus the central issue. and the best that can be said is

LEMMA 2.1. If $\psi$ is of type $k$ there exist $\delta>0$ and $C<\infty$ such that in the region $0 \leq x \leq x: \leq \cdots \leq x, \leq \delta$.

$$
\left|\frac{\partial y}{\partial x}\right| \geq C \prod_{1}^{n} x_{i}^{k_{i}+1-i} \cdot \prod_{i<j}\left(x_{j}-x_{i}\right)
$$

where $y=\sum_{i} \ell^{\prime}\left(x_{i}\right)$.

The proof is deferred until the end of the section. It will show that indeed no better estimate holds.

To justify the change of variables it suffices to show that in the region $0<x_{1}<$ $\cdots<x_{n} \leq \delta$. the map $x \rightarrow y(x)$ is injective with nonzero Jacobian. The Jacobian is nonzero by the lemma. so locally $x \rightarrow y(x)$ is a diffeomorphism. The hyperplanes $H_{t}=\left\{x: \sum x_{i}=t\right.$ and $\left.0<x_{1}<\cdots<x_{n} \leq \delta\right\}$ have disjoint images since $y_{1}=\sum x_{i}=t$. Since $x \rightarrow y$ is locally a diffeomorphism, its restriction to each $H_{t}$ is an immersion and the images of the $H_{t}$ locally foliate $\mathbf{R}^{n}$ into hypersurfaces. $H_{t}$ is connected (it is convex). so clearly the restriction to any $H_{t}$ of the map $x \rightarrow y$ is also injective: otherwise the image of $H_{t}$ would have to cross some other $H_{s}$ in the process of intersecting itself, a contradiction. The change of variables, then, is valicl.

The original restriction problem has now been reduced to the task of estimating certain positive multilinear operations. The best relevant estimates for these are provided by the next proposition. But first observe that the original problem is only equivalent to its reduction in the case $p^{\prime}=2 n$, by Plancherel's theorem; failure of the multilinear operations to be bounded does not settle the issue for other values of $p$.

Consider first the "ase of nondegenerate curves. For each $i, k_{i}=i-1$ so that $|\partial y / \partial x| \geq C \prod_{i<j \leq n}\left|x_{i}-x_{j}\right|$. Case (A) of Theorem 1.1 follows at once from the following proposition. (If $p, q$ are the exponents in the theorem and $\bar{p}$ of the proposition. they are related by $\bar{p}=r^{-1} q^{\prime}, r^{-1}=1-n\left(1-p^{-1}\right)$.) 
PROPOSITION 2.2. If $0 \leq \gamma$ then

$$
\int \prod_{i=1}^{n} f\left(x_{i}\right) \prod_{i<j \leq n}\left|x_{i}-x_{j}\right|^{-\gamma} d x_{1} \cdots d x_{n} \leq C\|f\|_{p}^{n},
$$

for all $f$, if and only if $\gamma<2 / n, 1 \leq p<n$ and $p^{-1}+\gamma(n-1) / 2=1$.

The restriction $p^{-1}+\gamma(n-1) / 2=1$ is mandated by homogeneity considerations, while if $\gamma \geq 2 / n$ and $f(x)=\chi_{|x| \leq 1}$ the integral with respect to $d x_{1}, \ldots, d x_{n-1}$ is infinite for each $\left|x_{n}\right| \leq 1$.

We will show that for any functions $f_{1}, \ldots, f_{n}, g_{1,2}, g_{1,3}, \ldots, g_{n-1, n}$. $(2.1)$

$$
\begin{aligned}
T\left(f_{1}, \ldots, f_{n} ; g_{1,2}, \ldots, g_{n-1, n}\right) & \equiv \int \prod_{1}^{n} f_{k}\left(x_{k}\right) \prod_{i<j} g_{i j}\left(x_{i}-x_{j}\right) d x_{1} \cdots d x_{n} \\
& \leq C \prod\left\|f_{k}\right\|_{p} \prod_{\left\|g_{i, j}\right\|_{q, \infty}}
\end{aligned}
$$

whenever $p^{-1}+\frac{1}{2}(n-1) q^{-1}=1$ and $1 \leq p<n$, where $\|\cdot\|_{q, r}$ denotes the usual Lorentz norm. By the Brascamp-Lieb-Luttinger rearrangement theorem this is actually no stronger than Proposition 2.1. What follows is one of several possible proofs of (2.1); for others see [4] and Remark 2 below.

Let $S_{n}$ denote the permutation group on $n$ letters and let $Q=[0,1]^{n} \times[0,1]^{\frac{1}{2} n(n-1)}$ $\subset \mathbf{R}^{m}$, where $m=n+\frac{1}{2} n(n-1)$. $S_{n}$ acts naturally on $Q$ by $\sigma\left(p^{-1} ; q^{-1}\right)=$ $\left(p_{\sigma(1)}^{-1}, \ldots, p_{\sigma(n)}^{-1} ; q_{\sigma(1), \sigma(2)}^{-1}, \ldots\right)$. We write $p^{-1}=\left(p^{-1}, \ldots, p_{n}^{-1}\right), f$ for $\left(f_{1}, \ldots, f_{n}\right)$, and so on. Let $G$ be the set of all $P=\left(p^{-1}, q^{-1}\right) \in Q$ for which

$$
T(f, g) \leq C(P) \prod\left\|f_{k}\right\|_{p_{k}} \prod\left\|g_{i, j}\right\|_{q_{i, j}}
$$

for all $f, g$. Let $H=\left\{P \in \mathbf{R}^{n}: \sum p_{k}^{-1}+\sum q_{i, j}^{-1}=n\right\}$. By homogeneity $G \subset H$. Therefore when we speak of an interior point of $G$, we mean interior with respect to the topology of $H$.

The proof is in two steps: first it will be shown that many vertices of $Q$ lie in $G$, just because of Fubini's theorem. Then these will be used as endpoints in a multilinear interpolation procedure. First of all $a=(1, \ldots, 1 ; 0, \ldots, 0)$ certainly is in $G$. So is the vertex $P_{0}$ with $p_{1}=1$, all other $p_{k}=\infty, q_{i, i+1}=1$ for each $i$ and $q_{i, j}=\infty$ if $j \neq i+1 ; P_{0}$ lies in $G$ by Fubini. Now $G$ is invariant under the natural action of $S_{n}$, so $\sigma\left(P_{0}\right) \in G$ for all $\sigma \in S_{n}$. Let $V=\left\{\sigma\left(P_{0}\right): \sigma \in S_{n}\right\}$, and let $b$ be the average of all points in $V$. By symmetry $b=\left(\frac{1}{n}, \ldots, \frac{1}{n} ; \frac{2}{n}, \ldots, \frac{2}{n}\right)$. $G$ coincides with its convex hull by (the proof of) the Riesz-Thörin interpolation theorem, so $b \in G$, and so is every point on the line segment $l$ joining $a$ to $b$. This gives (2.1) with the weak $L^{q}$ norms replaced by strong-type norms.

We claim that each point in the interior of $l$ lies in the interior of $G$. This granted, (2.1) follows by purely formal arguments and interpolation. For given any $k$ and $i, j$ we may view $T(f, g)$ as the result of applying a linear operator to $f_{k}$ and testing the outcome against $g_{i, j}$; all other functions $f_{r}, g_{s, t}$ are fixed and regarded as part of the operator. Interpolating by the real method then yields

$$
T(f, g) \leq C\left\|f_{k}\right\|_{p_{k}, 1}\left\|g_{i, j}\right\|_{q_{i, j}, \infty} \prod_{r \neq k}\left\|f_{r}\right\|_{p_{r}} \prod_{(s, t) \neq(i, j)}\left\|g_{s, t}\right\|_{q_{s, t}},
$$


for all $\left(p^{-1} \cdot q^{-1}\right)$ in the interior of $G$. Repeating this procedure with $k$ fixed and all possible choices of $i, j$ leads to

$$
T(f, g) \leq C\left\|f_{k}\right\|_{p_{k} .1} \prod_{r \neq k}\left\|f_{r}\right\|_{p_{r}} \prod_{s, t}\left\|g_{s, t}\right\|_{q_{s, t}, \infty},
$$

again for all $\left(p^{-1}, q^{-1}\right)$ in the interior of $G$. Now fix all $g_{s, t}$ for the remainder of the argument. Repeat the above process, with all functions fixed except $f_{k}$ and $f_{l}$, for some choice of $l \neq k$. Doing this for each $l \neq k$ gives

$$
T(f, g) \leq C\left\|f_{k}\right\|_{p_{k}, 1} \prod_{l \neq k}\left\|f_{l}\right\|_{p_{l}, \infty} \prod_{s, t}\left\|g_{s, t}\right\|_{q_{s, t}, \infty} .
$$

The final step is to interpolate yet again, using the fact that $\left(L^{p, r_{0}}, L^{p, r_{1}}\right)_{\theta, r}=$ $L^{p . r}$ if $r^{-1}=(1-\theta) r_{0}^{-1}+\theta r_{1}^{-1}$ and $0<\theta<1,1<p<\infty$; this is a special case of Theorem 5.2.4 of $[\mathbf{1}]$. Alternately freezing all but two of the $f$ 's, proceeding as above and finally restricting attention to $l$ we find that

$$
T(f, g) \leq C \prod\left\|f_{k}\right\|_{p, n} \prod\left\|g_{i, j}\right\|_{q, \infty}
$$

whenever $p^{-1}+\frac{1}{2}(n-1) q^{-1}=1$ and $1<p<n$. This is stronger than $(2.1)$; the appearance of the $L^{p, n}$ norms is a general feature of this type of argument for $n$-linear operations.

It remains only to verify the claim that each interior point of $l$ lies in the interior of $G$. Such a point may be expressed as a convex linear combination of $a$ and the points of $V$, in which all coefficients are nonzero. Hence it suffices to show that the linear span of $V-a=\{P-a: P \in V\}$ is the entire subspace $H-a$. Suppose $\nu \in \mathbf{R}^{m}$ were orthogonal to $V-a$. Then $\nu$ would be orthogonal to the difference of any two points of $V$. Let $P_{0}$ be as above, and let $P_{1}$ have the same coordinates except $q_{n-2, n-1}=\infty$ and $q_{n-2, n}=1$. $P_{0}, P_{1} \in V$ and all coordinates of $P_{0}-P_{1}$ are 0 . except that $q_{n-2, n}=-1$ and $q_{n-2, n-1}=1$. Therefore the $n-2, n$ coordinate of $\nu$ equals the $n-2, n-1$ coordinate, and it follows from the invariance of $V$ under $S_{n}$ that $\nu$ is of the form $\left(u_{1}, \ldots, u_{n} ; w, \ldots, w\right)$. Since $\left\langle\nu, P_{0}-a\right\rangle=0$, $\sum_{1}^{n} u_{i}=u_{1}+(n-1) w$. As a result $u_{1}=\sum_{1}^{n} u_{i}-(n-1) w$, whence by symmetry $u_{1}=\cdots=u_{m} \equiv w$. All coordinates of $\nu$ being equal, $\nu$ is orthogonal to $H-a$. We have shown that $(V-a)^{\perp}=(H-a)^{\perp}$, so the proof is complete.

Case (B) of Theorem 1.1 follows in the same way from Lemma 2.1 and

Proposition 2.3. Suppose $0<\gamma$ and $0=\beta_{1}<\beta_{2}<\cdots<\beta_{n}$. Let

$$
K(x)=\prod_{i<j}\left(x_{j}-x_{i}\right)^{-\gamma} \prod x_{i}^{-\beta_{i}} \chi_{0 \leq x_{1} \leq \cdots \leq x_{n}<\infty}
$$

Then

if and only if $\gamma<2 / n$ and either

$$
\int \prod f\left(x_{i}\right) K(x) d x \leq c\|f\|_{p}^{n}
$$

$$
1 \leq p \leq n \quad \text { and } \quad p^{-1}+\frac{1}{2}(n-1) \gamma+n^{-1} \sum \beta_{i}=1,
$$

or

$$
\begin{aligned}
& n<p \leq \infty, \quad p^{-1}+\frac{1}{2}(n-1) \gamma+n^{-1} \sum \beta_{i}<1 \text { and } \\
& f \text { is supported in a fixed bounded subset of }[0, \infty) .
\end{aligned}
$$


The requirement $p^{-1}+\frac{1}{2}(n-1) \gamma+n^{-1} \sum \beta_{i}=1$ follows from homogeneity. If $p>n$ and $p^{-1}+\frac{1}{2}(n-1) \gamma+n^{-1} \sum \beta_{i} \geq 1$, let

$$
f(t)=|t|^{-1 / p}|\log t|^{-1 / n} \chi_{0 \leq t \leq 1 / 2}
$$

In the region $0<x_{1} \leq \cdots \leq x_{n}$ and $x_{n} \leq 2 x_{1}, K(x) \geq C|x|^{-n(1-1 / p)}, f \in L^{p}$, yet

$$
\int \prod f\left(x_{i}\right) K(x) d x \geq C \int_{\substack{0<x_{1} \leq \ldots \leq x_{n} \leq 1 \\ x_{n} \leq 2 x_{1}}}|x|^{-n}|\log | x||^{-1} d x=\infty .
$$

If $n<p$ but $f$ is not required to have support in a fixed bounded set, then by homogeneity $p^{-1}+\frac{1}{2}(n-1) \gamma+n^{-1} \sum \beta_{i}$ must be 1 , which we have just seen cannot be.

PROOF. It suffices to treat the case $\beta_{1}=\cdots=\beta_{n}>0$; this dominates the general case because of the restriction $x_{i} \leq x_{i+1}$. But now that $\beta_{i}=\cdots=\beta_{n}$, the result is still true without that restriction, so we drop it and consider

$$
S\left(f_{1}, \ldots, f_{n}\right)=\int_{\mathbf{R}^{n}} \prod_{i} f_{i}\left(x_{i}\right) \prod_{i<j}\left|x_{i}-x_{j}\right|^{-\gamma} \prod_{i}\left|x_{i}\right|^{-\beta} d x .
$$

We may assume all the $f_{i}$ are nonnegative. We claim that if $1 \leq p \leq n, p^{-1}+$ $\frac{1}{2}(n-1) \gamma+\beta=1$ and $0<\gamma, \beta$ then

$$
S\left(f_{1}, \ldots, f_{n}\right) \leq C\left\|f_{1}\right\|_{p .1} \prod_{2}^{n}\left\|f_{i}\right\|_{p . \infty} .
$$

Let $f^{*}$ denote a symmetric nonincreasing rearrangement of $f$. By the BrascampLieb-Luttinger rearrangement inequality $[\mathbf{2}]$,

$$
S\left(f_{1}, \ldots, f_{n}\right) \leq S\left(f_{1}^{*}, \ldots, f_{n}^{*}\right) .
$$

To verify $(2.3)$, then, it is enough to check it when $f_{i}(t)=|t|^{-1 / p}$ for all $i \geq 2$. and $f_{1} \in L^{p, 1}$. By definition of $L^{p, 1}$ we may assume that $f_{1}$ is the characteristic function of a set, which by the rearrangement principle may be taken to be an interval centered at the origin. Finally, by homogeneity, it suffices to check the single instance $f_{1}(t)=\chi_{|t| \leq 1}$. Let

$$
F\left(x_{1}\right)=\left|x_{1}\right|^{-\beta} \int \prod_{2}^{n}\left|x_{i}\right|^{-\beta-1 / p} \prod_{1 \leq i<j}\left|x_{i}-x_{j}\right|^{-\gamma} d x_{2} \cdots d x_{n} .
$$

$F\left(x_{1}\right)=F(1)\left|x_{1}\right|^{-1 / q}$ where $q=p^{\prime}$. To see that $F(1)<\infty$, let

$$
g(t)=|t|^{-\beta-1 / p}|t-1|^{-\gamma}
$$

and specify an exponent $r$ by $r^{-1}+\frac{1}{2}(n-2) \gamma=1$. Then $1<r<n-1$ and $g \in L^{r}$. Indeed, there is no problem at infinity since

$$
r\left(\beta+p^{-1}+\gamma\right)>1 \Leftrightarrow 0<\beta+p^{-1}+\gamma-r^{-1}=\beta+p^{-1}+\frac{1}{2} n \gamma-1=\frac{1}{2} \gamma .
$$

Locally $r \gamma<1$ since

$$
r^{-1}=1-\frac{1}{2} n \gamma+\gamma>\gamma
$$

and $r\left(\beta+p^{-1}\right)<1$ since

$$
r^{-1}-\beta-p^{-1}=1-\frac{1}{2}(n-1) \gamma-\beta-p^{-1}+\frac{1}{2} \gamma=\frac{1}{2} \gamma>0 .
$$


Trivially $\gamma<2 /(n-1)$, so by Proposition 2.2

$$
\int \prod_{2}^{n} g\left(x_{i}\right) \prod_{1<i<j}\left|x_{i}-x_{j}\right|^{-\gamma} d x_{2} \cdots d x_{n} \leq C\|g\|_{r}^{n-1}<\infty .
$$

So (2.3) holds. But that $S\left(f_{1}, \ldots, f_{n}\right) \leq C \prod\left\|f_{i}\right\|_{p}$ now follows at once by interpolating as in the first step of the proof of Proposition 2.2; again the $L^{p}$ norm can be replaced with the Lorentz $L^{p, n}$ norm.

The case $p>n$ follows at once. For specify $\delta>0$ by $n^{-1}=p^{-1}+\delta$. It is no loss of generality to assume that the fixed bounded set on which $f$ is supported is $\{|t| \leq 1\}$. Then for any $\varepsilon>0, g(t) \equiv|t|^{-\delta+\varepsilon} f(t) \in L^{n}$.

$$
S(f, \ldots, f)=\int \prod g\left(x_{i}\right) \prod\left|x_{i}-x_{j}\right|^{-\gamma} \prod\left|x_{i}\right|^{-\beta+\delta-\varepsilon} d x .
$$

In order to apply the case $p=n$ we must check that there exists $\varepsilon>0$ such that $n^{-1}+\frac{1}{2}(n-1) \gamma+\beta+\varepsilon-\delta=1$ and $\beta+\varepsilon-\delta>0$.

$$
n^{-1}+\frac{1}{2}(n-1) \gamma+\beta+\varepsilon-\delta=p^{-1}+\frac{1}{2}(n-1) \gamma+\beta+\varepsilon,
$$

so $\varepsilon$ is determined, and

$$
\beta+\varepsilon-\delta=1-n^{-1}-\frac{1}{2}(n-1) \gamma>1-n^{-1}-\frac{1}{2}(n-1) 2 n^{-1}>0 .
$$

Therefore

$$
S\left(f_{1}, \ldots, f_{n}\right) \leq C\|g\|_{n}^{n} \leq C\|f\|_{p}^{n} .
$$

The deduction of Corollary 1.2 from these propositions is the same as that of the theorem; the change of variables remains valid in the homogeneous noncompact case.

REMARKS. 1. Proposition 2.3 has not been stated under the sharpest possible hypotheses; for instance it holds when $p=n, \gamma=\beta_{1}=0$ and $\beta_{2}=\cdots=\beta_{n}=1$. This case is a multilinear variant of Hardy's inequality

$$
\left\|y^{-1} \int_{0}^{y}|f(x)| d x\right\|_{p} \leq C\|f\|_{p}, \quad 1<p \leq \infty .
$$

2. There is no need to appeal to Proposition 2.2 in the proof; instead just proceed by induction. The argument also establishes Proposition 2.2 itself.

3 . It is possible to give a more tedious proof which does not appeal to the rearrangement principle. First one shows that when $n^{-1}+\frac{1}{2}(n-1)+\beta=1$,

$$
\int K(x) \prod f_{i}\left(x_{i}\right) d x \leq\left\|f_{k}\right\|_{q}\left\|f_{n}\right\|_{p} \prod_{i \neq k, n}\left\|f_{i}\right\|_{\infty}
$$

whenever $q=p^{\prime}, 1<p \leq \infty$, and $k \leq n-1$; this may be reduced to a variant of the above inequality of Hardy. The result follows from an appropriate interpolation first when $p=n$, then for all $1 \leq p \leq n$.

4. A "singular integral" version of Proposition 2.2 is discussed in $§ 5$. Different multilinear "fractional integral" operators are treated in $[\mathbf{5}, \mathbf{7}, \mathbf{8}]$. 
The proof of Lemma 2.1 rests on

FACT (Weyl [20, pp. 200-201]). Let $0 \leq k_{1}<\cdots<k_{n}$ be distinct integers. Let $\operatorname{det}\left(k_{1}, \ldots, k_{n}\right)$ be the determinant of the $n \times n$ matrix whose $i, j$ entry is $x_{i}^{k_{j}}$. Then all coefficients of the homogeneous symmetric polynomial

$$
D(k)(x)=\frac{\operatorname{det}\left(k_{1}, \ldots, k_{n}\right)}{\operatorname{det}(0,1, \ldots, n-1)}=\frac{\operatorname{det}\left(k_{1}, \ldots, k_{n}\right)(x)}{\prod_{i<j \leq n}\left(x_{j}-x_{i}\right)}
$$

are nonnegative integers.

PROOF OF LEMMA 2.1. Suppose $\psi^{\prime}(t)=\left(1, \phi_{2}(t), \ldots, \phi_{n}(t)\right)$ where $\phi_{i}(t)=$ $t^{k_{i}}(1+O(t))$. Let

$$
\begin{aligned}
F(x) & =\operatorname{det}\left(\phi_{i}\left(x_{j}\right)\right) / \prod_{i<j}\left(x_{j}-x_{i}\right)-D(k)(x) \\
& =\left(\operatorname{det}\left(\phi_{i}\left(x_{j}\right)\right)-\operatorname{det}\left(x_{i}^{k_{j}}\right)\right)\left(\prod_{i<j}\left(x_{j}-x_{i}\right)\right)^{-1} .
\end{aligned}
$$

We claim that for all $x_{1}, \ldots, x_{n} \geq 0,|F(x)| \leq C|x| D(k)(x)$. In order to establish this in the case where the $\phi_{i}$ are polynomials it is certainly enough to show that for each monomial $x^{\alpha}$ in the Taylor expansion (about the origin) of $F$, after rearranging the indices so that $\alpha_{i} \leq \alpha_{j}$ whenever $i<j$, we have, for each $1 \leq l \leq n$,

$$
\sum_{i \leq l} \alpha_{i} \geq \sum_{i \leq l}\left(k_{i}-i+1\right) \text {. }
$$

For $D(k)(x) \geq \prod_{1}^{n} x_{i}^{k_{i}-i+1}$ by the fact quoted above, since it is easily computed that this monomial actually appears in $D(k)$. When the $\phi_{i}$ are not polynomials this still suffices by Taylor's theorem; we leave the details to the reader.

Suppose there exists a monomial $x^{\alpha}$ (with $\alpha_{i} \leq \alpha_{j}$ whenever $i<j$ ) in the Taylor expansion of $F$ and an $l$ for which the "defect" $\sum_{i \leq l}\left(k_{i}-i+1\right)-\sum_{i \leq l} \alpha_{i}$ is positive. Only finitely many terms in the Taylor expansions of the $\phi_{k}$ could possibly give rise to any fixed term $x^{\alpha}$ in $F$, so it suffices to assume that all the $\phi_{i}$ are polynomials. Then choose $\alpha$ and $l$ for which the defect is maximal. In the product $F(x) \prod_{i<j}\left(x_{j}-x_{i}\right)$, one of the terms to which $x^{\alpha}$ gives rise is $\prod x_{i}^{\alpha_{i}+i-1}$. Since $x^{\alpha}$ has maximal defect, no other term of the product could cancel this one out. But clearly no such term $\prod x_{i}^{\alpha_{i}+i-1}$ arises in

$$
F(x) \prod_{i<j}\left(x_{j}-x_{i}\right)=\sum_{\sigma \in S_{n}} \operatorname{sgn}(\sigma) \prod \phi_{j}\left(x_{\sigma(j)}\right),
$$

so no $x^{\alpha}$ with positive defect could occur with nonzero coefficient in the Taylor expansion of $F$.

3. Codimension 2 in $\mathbf{R}^{4}$. For curves in $\mathbf{R}^{n}$ there is a natural notion of nondegeneracy from the point of view of curvature, namely the condition in hypothesis (A) of Theorem 1.1. From the point of view of the restriction problem for the Fourier transform, the natural notion of nondegeneracy is that (1.1) should hold with $\frac{1}{2} n(n+1) q=p^{\prime}$ (for $q$ in some range about which we will not be fussy). Then Theorem 1.1(A) asserts precisely that these two notions coincide. For manifolds of codimension one the situation is analogous: nonvanishing scalar curvature is the 
natural geometric condition, and it is equivalent to the validity of (1.1) with a certain optimal exponent $p=p(q)$ for all $q$ in a certain range. (This is a combination of results of Greenleaf [11], Knapp, Stein and Tomas [19].) Therefore it is natural to ask for a generalization to the intermediate cases. In the author's previous work [4] this question was studied in codimension two. A curvature condition of an unexpected type was shown to be necessary for (1.1) to hold with optimal $p$, and to be sufficient under either of two auxiliary hypotheses. We conjectured that it is in fact sufficient by itself. The case $n=4$ is exceptional since then the dimension of the submanifold divides the dimension of the ambient space, so that the methods of the preceding sections still apply. This enables us to prove the conjecture in this special case.

To accomplish this suppose that $M \subset \mathbf{R}^{4}$ is a smooth submanifold of codimension two. We work only in some small neighborhood of a given point $0 \in M$, so parametrize $M=\left\{(x, y ; \psi(x, y)):(x, y) \in U \subset \mathbf{R}^{2}\right\}$ where $\psi: U \rightarrow \mathbf{R}^{2}, 0 \in U$, $\nabla \psi(0)=0$ and $\psi(0)=0$. $M$ is equipped with a smooth measure $d \sigma=\phi(x, y) d x d y$, where $\phi \in C_{0}^{\infty}(U)$ and $\phi(0) \neq 0, \phi \geq 0$. It is known (Prestini [14]) that the restriction estimate

$$
\int_{M}|\hat{f}(\xi)|^{q} d \sigma \leq C\|f\|_{p}^{q} \quad \text { for all } f \in S\left(\mathbf{R}^{4}\right)
$$

can hold only if $3 q \leq p^{\prime}$. This section's main result, Theorem 3.2 , characterizes geometrically those $M$ for which (3.1) holds for some measure $\sigma$ nonvanishing at the given point $0 \in M$, with the best possible value of $p\left((3 q)^{\prime}\right)$ for $q$ in a certain range. Thus the following definition should be regarded as the correct geometric notion of nondegeneracy for manifolds of dimension two in $\mathbf{R}^{4}$ as far as the restriction problem is concerned. (The general study of the degenerate case, analogous to case (B) of Theorem 1.1, is more complicated than for curves, and we do not venture into those waters.)

Parametrize the set of unit vectors in $\mathbf{R}^{4}$ normal to $M$ at 0 by $\theta \in[0,2 \pi] ; \theta$ will denote either a unit vector in $\mathbf{R}^{4}$ or the corresponding real number, depending on the context.

DEFINITION 3.1. If $M=\left\{(x ; \psi(x)): x \in U \subset \mathbf{R}^{2}\right\}$ as above with $0 \in U$ and $\nabla \psi(0)=0$, then $M$ is nondegenerate at 0 if

$$
\left.\operatorname{det} D_{x}^{2}\left(\left\langle\psi(x), \theta_{0}\right\rangle\right)\right|_{x=0}=\left.0 \Rightarrow \frac{d}{d \theta}\left(\left.\operatorname{det} D_{x}^{2}\langle\psi(x), \theta\rangle\right|_{x=0}\right)\right|_{\theta=\theta_{0}} \neq 0 .
$$

Here $D_{x}^{2}$ denotes the Hessian matrix of second partial derivatives with respect to $x$ and of course det denotes the determinant. An example of a nondegenerate manifold is $\left(x, y, x^{2}, y^{2}\right)$. There is a natural geometric interpretation: each normal vector $\theta$ determines, together with the two-plane tangent to $M$ at 0 , a hyperplane $P(\theta)$. det $\left.D_{x}^{2}\langle\psi(x), \theta\rangle\right|_{x=0}$ measures the scalar curvature (up to a nonvanishing factor) of the codimension one manifold in $\mathbf{R}^{3}$ obtained by projecting $M$ locally onto $P(\theta)$, and the assumption is that as a function of $\theta$, this scalar curvature has no zeroes of order greater than one. This interpretation plays no role in our arguments.

THEOREM 3.2. Suppose that $M \subset \mathbf{R}^{4}$ is smooth of codimension two and $0 \in$ $M$. Then the following are equivalent:

(3.2) $M$ is nondegenerate at 0. 
(3.3) There exist a smooth measure $\sigma$ on $M$, nonvanishing at 0 , and an exponent $p>1$ such that $\|\hat{f}\|_{L^{q}(M, \sigma)} \leq C\|f\|_{L^{p}\left(\mathbf{R}^{4}\right)}$ for all $f \in S$, where $3 q=p^{\prime}$.

(3.4) The inequality in (3.3) holds for all $1 \leq p<4 / 3$ and $3 q=p^{\prime}$.

In fact the proof will show that (3.3) implies (3.2) if the assumption $3 q=p^{\prime}$ is weakened to $3 q \geq p^{\prime}-\delta$ for a certain $\delta=\delta(q)>0$.

The proof of the following is an exercise in linear algebra and is left to the reader.

LEMMA 3.3. If $M=\{(x, y ; \psi(x, y))\}$ is nondegenerate at 0 then it is possible to make a linear transformation of coordinates in $\mathbf{R}^{4}$ so that either

$$
\psi(x, y)=\left(x^{2}-y^{2}, x y\right)+O(x, y)^{3}
$$

or

$$
\psi(x, y)=\left(x^{2}, y^{2}\right)+O(x, y)^{3} .
$$

If $M$ is degenerate at 0 then coordinates may be introduced in which

$$
\psi(x, y)=\left(x^{2}, 0\right)+O(x, y)^{3} \quad \text { or } \quad \psi(x, y)=(0,0)+O(x, y)^{3} .
$$

The definition of nondegeneracy and a rather more complicated canonical form for degenerate manifolds of codimension two in higher dimensions may be found in $[4, \S 3]$.

Proof OF THEOREM 3.2. (3.4) implies (3.3) tautologously. If (3.2) fails, $\psi$ may be written as $\left(x^{2}, 0\right)+O(x, y)^{3}$ or in an even more degenerate form. In the first case consider, for $\varepsilon>0$ small, the function $f_{\varepsilon}$ whose Fourier transform is the characteristic function of the set where $|x, y| \leq \varepsilon,|u| \leq \varepsilon^{2}$ and $|v| \leq \varepsilon^{3}$ with coordinates $(x, y ; u, v)$ in $\mathbf{R}^{4}$. $\left\|\hat{f}_{\varepsilon}\right\|_{L^{q}(M, \sigma)} \geq C \varepsilon^{2 / q}$ for $\varepsilon$ small, while $\left\|f_{\varepsilon}\right\|_{p}=$ $C \varepsilon^{7(1-1 / p)}$. Thus $2 / q \geq 7(1-1 / p)$, so (3.3) fails also. The more degenerate case follows by the same argument.

To prove that (3.2) implies (3.4) we use the Hausdorff-Young inequality as in $\S 2$ to reduce the study of the adjoint of the restriction operator to estimates for certain positive operations, which in the present situation are linear rather than multilinear. The details of this reduction may be found in Prestini [14] and are omitted here. However it is necessary to compute explicitly the Jacobian of the resulting change of variables.

Adopt coordinates $z=(x, y)$ on $\mathbf{R}^{2}$ and let $\psi(z)=(P(z), Q(z)) \in \mathbf{R}^{2}$. Consider the change of coordinates in $\mathbf{R}^{4}$ defined by

$$
w=\left(z_{1}+z_{2} ; P\left(z_{1}\right)+P\left(z_{2}\right), Q\left(z_{1}\right)+Q\left(z_{2}\right)\right) .
$$

Then

$$
\begin{aligned}
\frac{\partial w}{\partial\left(z_{1}, z_{2}\right)}= & \left(\frac{\partial P}{\partial x_{2}} \frac{\partial Q}{\partial y_{2}}-\frac{\partial P}{\partial y_{2}} \frac{\partial Q}{\partial x_{2}}\right)+\left(\frac{\partial P}{\partial y_{1}} \frac{\partial Q}{\partial x_{2}}-\frac{\partial P}{\partial x_{2}} \frac{\partial Q}{\partial y_{1}}\right) \\
& -\left(\frac{\partial P}{\partial x_{1}} \frac{\partial Q}{\partial y_{2}}-\frac{\partial P}{\partial y_{2}} \frac{\partial Q}{\partial x_{1}}\right)+\left(\frac{\partial P}{\partial x_{1}} \frac{\partial Q}{\partial y_{1}}-\frac{\partial P}{\partial y_{1}} \frac{\partial Q}{\partial x_{1}}\right) ;
\end{aligned}
$$

it is understood that $\partial P / \partial x_{i}$ is a function of $z_{i}$ alone, and so on. When $(P(z), Q(z))$ $=\left(x^{2}, y^{2}\right)+O(z)^{3}$,

$$
\frac{\partial w}{\partial\left(z_{1}, z_{2}\right)}=\left(x_{1}-x_{2}\right)^{2}-\left(y_{1}-y_{2}\right)^{2}+O(z) O\left(z_{1}-z_{2}\right)^{2}
$$


Assume for the remainder of this paragraph that $\left|z_{1}\right|,\left|z_{2}\right| \leq \delta$ where $\delta>0$ is a small number. Fix $z_{2}$ and let $u=x_{1}-x_{2}, v=y_{1}-y_{2}$, and $F(u, v)=\partial w / \partial\left(z_{1}, z_{2}\right)$. By the Morse lemma there are coordinates $\left(u^{\prime}, v^{\prime}\right)$ with respect to which (locally) $F(u, v)=\left(u^{\prime}\right)^{2}-\left(v^{\prime}\right)^{2}=\left(u^{\prime}-v^{\prime}\right)\left(u^{\prime}+v^{\prime}\right)$. Furthermore $u^{\prime}=u+O\left(z_{2}\right) O(u, v)$ and $v^{\prime}=v+O\left(z_{2}\right) O(u, v)$. If $\delta$ is small enough the implicit function theorem gives

$$
\left|u^{\prime}-v^{\prime}\right| \sim|u-\xi(v)| \text { and }\left|u^{\prime}+v^{\prime}\right| \sim|u-\eta(v)|
$$

where $\xi, \eta$ are smooth and also depend smoothly on the parameter $z_{2}$, and, moreover, $|\xi(v)-\eta(v)| \sim 2|v|$.

Recall that on any $\sigma$-finite measure space

$$
\left\|\int F(s) A(s, t) d s\right\|_{q} \leq C\|F\|_{p}\left(\sup _{s}\|A(s, \cdot)\|_{r, \infty}+\sup _{t}\|A(\cdot, t)\|_{r, \infty}\right)
$$

provided $1 / q=1 / p+1 / r-1$ and $1<p, q, r<\infty$ (Strichartz [17]). Together with a piggyback procedure this gives

LEMMA 3.4. Suppose $\psi$ has the form (3.5) or (3.6), and $w\left(z_{1}, z_{2}\right)$ is defined by (3.7). There exists $\delta>0$ such that given any $0<\gamma<1, r \gamma=1,1 / q=1 / p+1 / r-1$ and $1<p, q, r<\infty$, then

$$
\left\|f\left(z_{1}\right) K\left(z_{1}, z_{2}\right) d z_{1}\right\|_{q} \leq C\|f\|_{p}
$$

where

$$
K\left(z_{1}, z_{2}\right)=\left|\partial w / \partial\left(z_{1}, z_{2}\right)\right|^{-\gamma} \chi_{\left|z_{1}\right|,\left|z_{2}\right| \leq \delta}
$$

PROOF. First suppose we are in the situation of (3.6). Let

$$
S f\left(y_{1}, x_{2}, y_{2}\right)=\int f\left(x_{1}, y_{1}\right) K\left(x_{1}, y_{1}, x_{2}, y_{2}\right) d x_{1} \text {. }
$$

By (3.8)

$$
\left(\int\left|S f\left(y_{1}, x_{2}, y_{2}\right)\right|^{q} d x_{2}\right)^{1 / q} \leq C\left(\int\left|f\left(x_{1}, y_{1}\right)\right|^{p} d x_{1}\right)^{1 / p} A\left(y_{1}, y_{2}\right)
$$

where

$$
A\left(y_{1}, y_{2}\right)=\sup _{x_{1}}\left\|K\left(x_{1}, y_{1}, \cdot, y_{2}\right)\right\|_{r, \infty}+\sup _{x_{2}}\left\|K\left(\cdot, y_{1}, x_{2}, y_{2}\right)\right\|_{r, \infty}
$$

In order to apply (3.8) again with $F\left(y_{1}\right)=\left(\int\left|f\left(x_{1}, y_{1}\right)\right|^{p} d x_{1}\right)^{1 / p}$ we need to verify that

$$
\sup _{y_{1}}\left\|A\left(y_{1}, \cdot\right)\right\|_{r, \infty}+\sup _{y_{2}}\left\|A\left(\cdot, y_{2}\right)\right\|_{r, \infty} \leq C<\infty .
$$

In fact $A\left(y_{1}, y_{2}\right)=A\left(y_{2}, y_{1}\right)$ since $K$ is symmetric in $z_{1}, z_{2}$, so these last two expressions are identical.

$$
\begin{aligned}
\left\|A\left(\cdot, y_{2}\right)\right\|_{r, \infty}^{r}=\sup _{\lambda>0} \lambda^{-r} \mid\left\{y_{1}: \sup _{x_{1}}\left\|K\left(x_{1}, y_{1}, \cdot, y_{2}\right)\right\|_{r, \infty}\right. \\
\left.\quad+\sup _{x_{2}}\left\|K\left(\cdot, y_{1}, x_{2}, y_{2}\right)\right\|_{r, \infty}>\lambda\right\} \mid .
\end{aligned}
$$


For fixed $x_{2}, y_{2}$ we have found that

so

$$
K\left(x_{1}, y_{1}, x_{2}, y_{2}\right) \leq C\left|x_{1}-\left(x_{2}+\xi\left(y_{1}\right)\right)\right|^{-\gamma}\left|x_{1}-\left(x_{2}-\eta\left(y_{1}\right)\right)\right|^{-\gamma},
$$

$$
\left\|K\left(\cdot, y_{1}, x_{2}, y_{2}\right)\right\|_{r, \infty}^{r} \leq C\left|\xi\left(y_{1}\right)-\eta\left(y_{1}\right)\right|^{-1} \leq C\left|y_{1}\right|^{-1}
$$

where $C$ does not depend on $x_{2}, y_{2}$. This estimate holds for $\left\|K\left(x_{1}, y_{1}, \cdot, y_{2}\right)\right\|_{r, \infty}$ as well; to see this go back to the starting point

$$
\frac{\partial w}{\partial\left(z_{1}, z_{2}\right)}=\left(x_{1}-x_{2}\right)^{2}-\left(y_{1}-y_{2}\right)^{2}+O(z) O\left(x_{1}-x_{2}, y_{1}-y_{2}\right)
$$

freeze $x_{1}$ and $y_{2}$, and repeat all of the above arguments. Since this form for $\partial w / \partial\left(z_{1}, z_{2}\right)$ is invariant under interchanging $x_{1}$ and $x_{2}$, and is all we used to estimate $\left\|K\left(\cdot, y_{1}, x_{2}, y_{2}\right)\right\|_{r, \infty}$, the same result is obtained. Thus

$$
\left\|A\left(\cdot, y_{2}\right)\right\|_{r, \infty}^{r} \leq C \sup _{\lambda>0} \lambda^{-r}\left|\left\{y_{1}: 2 C\left|y_{1}\right|^{-1 / r}>\lambda\right\}\right| \leq C .
$$

By Minkowski's integral inequality

$$
\begin{aligned}
\left\|\int f\left(z_{1}\right) K\left(z_{1}, z_{2}\right) d z_{1}\right\|_{q}^{q} & =\int\left(\int S f\left(y_{1}, x_{2}, y_{2}\right) d y_{1}\right)^{q} d x_{2} d y_{2} \\
& \leq \int\left(\int\left(\int S f\left(y_{1}, x_{2}, y_{2}\right)^{q} d x_{2}\right)^{1 / q} d y_{1}\right)^{q} d y_{2} \\
& \leq C\left\|\int F\left(y_{1}\right) A\left(y_{1}, \cdot\right) d y_{1}\right\|_{q}^{q} \leq C\|F\|_{p}^{q} \leq C\|f\|_{p}^{q}
\end{aligned}
$$

by $(3.8)$ and $(3.9)$.

This concludes the proof in the case where $\psi$ satisfies (3.6). In case (3.5), one finds that

$$
\frac{\partial w}{\partial\left(z_{1}, z_{2}\right)}=2\left(x_{1}-x_{2}\right)^{2}+2\left(y_{1}-y_{2}\right)^{2}+O(z) O\left(z_{1}-z_{2}\right)^{2} \geq C\left|z_{1}-z_{2}\right|^{2},
$$

so that it is enough to apply well-known estimates for fractional integrals in $\mathbf{R}^{2}$.

4. A related theorem. In a sense nontrivial restriction theorems for the Fourier transform are made possible by the following negative result:

THEOREM 4.1. Suppose $h: \mathbf{R}^{n} \rightarrow \mathbf{R}^{n}$ is a $C^{2}$ diffeomorphism, and define a linear operator by

$$
(T f) \widehat{\urcorner}(\xi)=\hat{f}(h(\xi)) .
$$

If $T$ is bounded on $L^{p}$, then either $p=2$ or $h$ is a linear transformation plus a constant.

For suppose $n=2, h$ is not affine and $T$ is bounded on some $L^{p}, 1<p<4 / 3$. Then there exists $\psi:(-1,1) \rightarrow \mathbf{R}^{2}$ whose image is a curve with nonvanishing curvature, such that the image of $h \circ \psi$ is a straight line segment. Let $q=(3 p)^{\prime}$. Since restriction of the Fourier transform to the curve given by $\psi$ maps $L^{p}\left(\mathbf{R}^{2}\right)$ to $L^{q}$

$$
\int|\hat{f}(h \circ \psi(t))|^{q} d t=\int\left|(T f)^{\wedge}(\psi(t))\right|^{q} d t \leq C\|T f\|_{p}^{q} \leq C\|f\|_{p}^{q},
$$

which is absurd. 
This observation arose in a conversation between A. P. Calderón, P. W. Jones and the author; the following proof is adapted from an argument presented by Katznelson [13] for the Helson-Beurling theorem, which asserts (when $n=1$ ) that the same conclusion holds when $p=1$ even when $h$ is only assumed to be continuous. Notice that some regularity assumption on $h$ is needed when $1<p<\infty$, as shown by the example

$$
h(x)= \begin{cases}x & \text { if } x \leq 0 \\ 2 x & \text { if } x \geq 0\end{cases}
$$

when $n=1$. (Boundedness of $T$ follows from boundedness of the Hilbert transform.)

PROOF. To begin suppose that $n=1$ and $1 \leq p<2$. Assume merely that $h$ is $C^{1}$ and that there exists an interval $I$ on which $h^{\prime}$ is (strictly) monotone; if $h$ is $C^{2}$ and no such interval exists, then $h$ is linear. It would suffice to produce a function $f$ such that $\|f\|_{p},\|T f\|_{2} \leq 1$ and $\|T f\|_{\infty}$ is arbitrarily small. For then $\|T f\|_{p}^{p} \geq\|T f\|_{2}^{2}\|T f\|_{\infty}^{p-2}$ is as large as desired.

Choose $g \in C_{0}^{\infty}$, supported on such an inteval $I$ and not identically zero. For each $x \in \mathbf{R}$ let

$$
J(x, \varepsilon)=\left\{\xi \in I:\left|h^{\prime}(t)-x\right|<\varepsilon\right\} .
$$

By hypothesis $J(x, \varepsilon)$ is an interval, possibly empty, and $\sup _{x}|J(x, \varepsilon)| \rightarrow 0$ as $\varepsilon \rightarrow 0$. For a laige parameter $r$, define $f_{r}$ by $\hat{f}_{r}(\xi)=\exp (\operatorname{ir} \xi) g(\xi)$. For any $x \in \mathbf{R}$ and any $\rho>0$, writing $J=J\left(r^{-1} x, r^{-1} \rho\right)$ we have

$$
\left|T f_{r}(x)\right| \leq\|g\|_{\infty}|J|+\int_{I \backslash J} \exp (i(r h(\xi)-x \xi)) \cdot g(h(\xi)) d \xi .
$$

By integration by parts and the hypothesis on $h^{\prime}$ this is dominated by $C\left(|J|+\rho^{-1}\right)$. If $\rho=r^{1 / 2}$ both terms tend to 0 as $r \rightarrow \infty$.

When $p>2$ it is not sufficient to merely pass to the adjoint operator, since the Jacobian determinant of $h$, which might fail to be a multiplier on $L^{p}$, then arises. But if $h^{-1}$ satisfies the above hypothesis on some $I$, let

$$
\hat{f}_{r}(\xi)=\exp \left(i r h^{-1}(\xi)\right) g\left(h^{-1}(\xi)\right) .
$$

$f_{r} \rightarrow 0$ in $L^{\infty}$ as $r \rightarrow \infty$, and of course $\left\|f_{r}\right\|_{2}$ is independent of $r$. Hence $f_{r} \rightarrow 0$ in $L^{p}$ as $r \rightarrow \infty$. $T f_{r}(x)=f_{0}(x-r)$ has constant $L^{p}$ norm, so again $T$ is unbounded.

When $n>1$ it is only necessary to apply the same argument to $\hat{f}_{r}(\xi)=$ $\exp (i\langle\nu, h(\xi)\rangle) g(\xi)$ for some $\nu \in S^{n-1}$.

I am indebted to T. Wolff for calling my attention to Katznelson's treatment $[13]$.

5. Some multilinear singular integrals. Although Proposition 2.2 is false at the endpoint $\gamma=2 / n$, a deeper substitute does hold. We restrict our attention to an a priori inequality, and give only a sketch of the proof, ignoring all questions concerning the convergence and interpretation of various integrals (which should be interpreted as principal value integrals).

THEOREM 5.1. Suppose $\gamma=2 / n+i \tau, 0 \neq \tau \in \mathbf{R}$. Let

$$
T_{\gamma}\left(f_{1}, \ldots, f_{n}\right)=\int \prod_{i=1}^{n} f_{i}\left(x_{i}\right) \prod_{i<j \leq n}\left|x_{i}-x_{j}\right|^{-\gamma} d x_{1} \cdots d x_{n},
$$


where $x_{1}, \ldots, x_{n} \in \mathbf{R}$. Then for all $f_{1}, \ldots, f_{n} \in S$,

$$
\left|T_{\gamma}\left(f_{1}, \ldots, f_{n}\right)\right| \leq C\left(p_{1}, \ldots, p_{n}, \tau\right) \prod\left\|f_{j}\right\| p_{j}
$$

provided $\sum_{1}^{n} p_{j}^{-1}=1$ and $1<p_{j}$ for all $j$.

Proposition 2.2 follows: interpolate using the analytic family of operations $\gamma \rightarrow$ $T_{\gamma}$. However the argument given in $\S 2$ is more elementary and is useful in other contexts. The sketch of the proof is based on a powerful theorem due to G. David and J. Journé [6]:

THEOREM. Suppose $T: D(\mathbf{R}) \rightarrow D^{\prime}(\mathbf{R})$ is a linear operator,

$$
K \in L_{\mathrm{loc}}^{1}\left(\mathbf{R}^{2} \backslash\{x=y\}\right) \quad \text { and } \quad\langle T \phi, \psi\rangle=\int \phi(x) \psi(y) K(x, y) d x d y
$$

for all $\phi, \psi \in D$ with disjoint supports. Suppose

$$
\begin{gathered}
|K(x, y)| \leq B|x-y|^{-1} \\
\left|\partial_{x} K(x, y)\right|+\left|\partial_{y} K(x, y)\right| \leq B(x-y)^{-2} \quad \text { if } x \neq y
\end{gathered}
$$

and

$$
\left\|T\left(e^{i x \xi}\right)\right\|_{\mathrm{BMO}}+\left\|T^{*}\left(e^{i x \xi}\right)\right\|_{\mathrm{BMO}} \leq B \quad \text { for all } \xi \in \mathbf{R} .
$$

Then $\|T f\|_{p} \leq C(p) B\|f\|_{p}$ for all $1<p<\infty$ and $\|T f\|_{1} \leq C B\|f\|_{H^{1}}$ for all $f \in D$.

To obtain our inequality it suffices to show that

$$
\left|T_{\gamma}\left(f_{1}, \ldots, f_{n}\right)\right| \leq C(p, \tau)\left\|f_{1}\right\|_{p}\left\|f_{2}\right\|_{p^{\prime}} \prod_{3}^{n}\left\|f_{j}\right\|_{\infty}
$$

for all $1<p<\infty$. Fix $f_{3}, \ldots, f_{n}$ and set

$$
K\left(x_{1}, x_{2}\right)=\int \prod_{3}^{n} f_{j}\left(x_{j}\right) \prod_{1 \leq i<j \leq n}\left|x_{i}-x_{j}\right|^{-\gamma} d x_{3} \cdots d x_{n},
$$

so that formally

$$
T\left(f_{1}, \ldots, f_{n}\right)=\int f_{1}\left(x_{1}\right) f_{2}\left(x_{2}\right) K\left(x_{1}, x_{2}\right) d x_{1} d x_{2} .
$$

By the David-Journé characterization it suffices to show that

$$
\left|T\left(f_{1}, \exp \left(i x_{2} \xi\right), f_{3}, \ldots, f_{n}\right)\right| \leq C\left\|f_{1}\right\|_{H^{1}} \prod_{3}^{n}\left\|f_{j}\right\|_{\infty}
$$

for any $\xi \in \mathbf{R}$, since straightforward computation reveals that $K$ satisfies the first two hypotheses of their theorem with $B=C \prod_{3}^{n}\left\|f_{j}\right\|_{\infty}$.

Define a new kernel

$$
L\left(x_{1}, x_{3}\right)=\int e^{i \xi x_{2}} \prod_{j \neq 1,2,3} f_{j}\left(x_{j}\right) \prod_{1 \leq i<j \leq n}\left|x_{i}-x_{j}\right|^{-\gamma} \prod_{j \neq 1,3} d x_{j} .
$$


The situation is just as above, except that attention has been restricted to the case when $f_{2}$ is a character. Repeating the argument reduces our task to establishing $(5.1)$ in the case when $f_{2}, \ldots, f_{n}$ are all characters. Formally

$$
\int \prod_{2}^{n} \exp \left(i \xi_{j} x_{j}\right) \prod_{1 \leq i<j \leq n}\left|x_{i}-x_{j}\right|^{-\gamma} d x_{2} \cdots d x_{n}=\exp \left(i x_{1} \Sigma \xi_{j}\right) \hat{F}\left(\xi_{2}, \ldots, \xi_{n}\right)
$$

where

$$
F\left(x_{2}, \ldots, x_{n}\right)=\prod_{2}^{n}\left|x_{j}\right|^{-\gamma} \prod_{2 \leq i<j \leq n}\left|x_{i}-x_{j}\right|^{-\gamma} \quad \text { on } \mathbf{R}^{n-1} .
$$

$F$ is homogeneous of degree $-(n-1)-i \frac{1}{2} n(n-1) \tau$ and is integrable on the unit sphere, so $\hat{F}$ is bounded. Therefore (5.1) holds with $\|f\|_{H^{1}}$ replaced by $\|f\|_{L^{1}}$.

ADDED IN PROOF. Since this article was written further results concerning the restrictions of Fourier transforms to curves have been obtained by S. W. Drury and B. P. Marshall. Moreover I have learned that Theorem 4.1 is known, and is due to L. Hörmander (Acta Math. 104 (1960)).

\section{REFERENCES}

1. J. Bergh and J. Löfstrom, Interpolation spaces: an introduction, Springer-Verlag, New York, 1976.

2. H. J. Brascamp, E. H. Lieb and J. M. Luttinger, A general rearrangement inequality for multiple integrals, J. Funct. Anal. 17 (1974), 227-237.

3. L. Carleson and P. Sjölin, Oscillatory integrals and a multiplier problem for the disc, Studia Math. 44 (1972), 287299.

4. M. Christ, Restriction of the Fourier transform to submanifolds of low codimension, Ph.D. dissertation, Univ. of Chicago, Chicago, Ill., 1982.

5. _ Estimates for the $k$ plane transform, Indiana Univ. Math. J. (to appear).

6. G. David and J. Journé, A boundedness criterion for generalized Calderón-Zygmund operators (preprint).

7. S. W. Drury. Generalizations of Riesz potentials and $L^{p}$ estimates for certain $k$-plane transforms (preprint).

8. . $^{p}$ estimates for the $x$ ray transform, Illinois J. Math. 27 (1983), 125-129.

9. C. Fefferman, Inequalities for strongly singular convolution operators, Acta Math. 124 (1970), 9-36.

10. _ . The multiplier problem for the ball, Ann. of Math. (2) 94 (1971), 330-336.

11. A. Greenleaf, Principal curvature and harmonic analysis (preprint).

12. L. Hörmander, Oscillatory integrals and multipliers on $F L^{p}$, Ark. Mat. 11 (1973), 111.

13. Y. Katznelson, An introduction to harmonic analysis, Wiley, New York, 1968.

14. E. Prestini, Restriction theorems for the Fourier transform to some manifolds in $\mathbf{R}^{n}$, Proc. Sympos. Pure Math., Vol. 35, Part 1, Amer. Math. Soc., Providence, R.I., 1979, pp. 101 109.

15. A. Ruiz, On the restriction of Fourier transforms to curves, Conf. Harmonic Analysis in Honor of Antoni Zygmund (W. Beckner, et al., eds.), Vol. 1, Wadsworth, Belmont, Calif., 1983, pp. 186212.

16. P. Sjölin, Fourier multipliers and estimates of Fourier transforms of measures carried by smooth curves in $\mathbf{R}^{2}$, Studia Math. 51 (1974), 169182.

17. R. Strichartz. $L^{p}$ estimates for integral transforms, Trans. Amer. Math. Soc. 136 (1969), 33-50.

18. _ Restrictions of Fourier transforms to quadratic surfaces and decay of solutions of wave equations, Duke J. Math. 44 (1977), 705714.

19. P. A. Tomas, Restriction theorems for the Fourier transform, Proc. Sympos. Pure Math., Vol. 35, Part 1. Amer. Math. Soc., Providence, R.I., 1979, pp. 111114.

20. H. Weyl, The classical groups, Princeton Univ. Press. Princeton, N.J., 1946. 08544

Department of Mathematics. Princeton University, Princeton, New Jersey 